\title{
EFFECTIVENESS OF SIMILAR SETS OF POLYNOMIALS OF TWO COMPLEX VARIABLES IN POLYCYLINDERS AND IN FABER REGIONS
}

\author{
K.A.M. SAYYED \\ Department of Mathematics \\ University of Assiut \\ Assiut 71516, EGYPT \\ and

\section{M.S. METWALLY} \\ Department of Mathematics \\ Unıversity of Assiut \\ Assiut 71516, EGYPT
}

(Received November 16, 1995 and in revised form April 21, 1997)

\begin{abstract}
In the present paper we investigate the effectiveness of similar sets of polynomials of two complex variables in polycylinder and in Faber regions of the four dimensional space $E^{4}$
\end{abstract}

KEY WORDS AND PHRASES: Simple sets, similar sets, Faber polynomials, Faber regions, polycylinders.

1991 AMS SUBJECT CLASSIFICATION CODES: 32A15

\section{INTRODUCTION}

Let $\left\{p_{m, n}\left(z_{1}, z_{2}\right)\right\}$ be a basic set of polynomials of two complex variables, where

$$
p_{m, n}\left(z_{1}, z_{2}\right)=\sum_{i, j=0}^{m, n} p_{m, n ; ?, j} z_{1}^{2} z_{2}^{\jmath}
$$

The index of the last monomials in a given polynomial is called the degree of the polynomial and a set $\left\{p_{j}\left(z_{1}, z_{2}\right)\right\}$ of polynomials in which $j$ equals the degree of the polynomials $p_{j}\left(z_{1}, z_{2}\right)$ is called a simple set If the set of polynomials consists of a base, then every monomial $z_{1}^{m} z_{2}^{n}, m, n \geq 0$ admits a unique finite representation of the form

$$
z_{1}^{m} z_{2}^{n}=\sum_{i, j=0}^{m, n} \bar{p}_{m, n, 2, j} p_{\imath \jmath}\left(z_{1}, z_{2}\right)
$$

Suppose that the function $f\left(z_{1}, z_{2}\right)$ is regular in a neighborhood of the origin $(0,0)$, then it can be represented by the set of polynomials in some polycylinder $\bar{\Gamma}_{r_{1}, r_{2}}$ as follows $\left(\Gamma_{r_{1}, r_{2}} ; r_{k}>0, k=1,2\right.$ is the open connected set defined as follows $\Gamma_{r_{1}, r_{2}}=\left\{\left(z_{1}, z_{2}\right):\left|z_{k}\right|<r_{k}\right\}$ its closure is denoted by $\left.\bar{\Gamma}_{r_{1}, r_{2}}\right)$

$$
f\left(z_{1}, z_{2}\right)=\sum_{m, n=0}^{\infty} a_{m, n} z_{1}^{m} z_{2}^{n}=\sum_{m, n=0}^{\infty} a_{m, n} \sum_{j=0}^{\infty} \bar{p}_{m, n ; j} p_{n}\left(z_{1}, z_{2}\right) \sim \sum_{j=0}^{\infty} \prod^{\jmath} f(0,0) p_{\jmath}\left(z_{1}, z_{2}\right)
$$

where

$$
\prod^{\jmath} f(0,0)=\sum_{m, n=0}^{\infty} a_{m, n} p_{m, n,} .
$$

The basic set $\left\{p_{m, n}\left(z_{1}, z_{2}\right)\right\}$ of polynomials is said to represent the function $f\left(z_{1}, z_{2}\right)$ in the polycylinder $\bar{\Gamma}_{r_{1}, r_{2}}$, where the function is regular, if the last series in (1.3) converges uniformly to $f\left(z_{1}, z_{2}\right)$ in $\bar{\Gamma}_{r_{1}, r_{2}}$ If the basic set $\left\{p_{m, n}\left(z_{1}, z_{2}\right)\right\}$ represents in $\bar{\Gamma}_{r_{1}, r_{2}}$ every function which is regular there then the set is said to be effective in $\bar{\Gamma}_{r_{1}, r_{2}}$ 
The Cannon sum $\omega_{m, n}\left(r_{1}, r_{2}\right)$ and the Cannon function $\lambda\left(r_{1}, r_{2}\right)$ of the set $\left\{p_{m, n}\left(z_{1}, z_{2}\right)\right\}$ for the polycylinder $\bar{\Gamma}_{r_{1}, r_{2}}$ are defined as follows (c f. [3], pp 52, 55)

and

$$
\omega_{m, n}\left(r_{1}, r_{2}\right)=r_{1}^{n} r_{2}^{m} \sum_{i, j=0}^{m, n}\left|\bar{p}_{m, n, \imath, j}\right| M\left(p_{\imath \jmath} ; r_{1}, r_{2}\right)
$$

where

$$
\lambda\left(r_{1}, r_{2}\right)=\limsup _{m+n \rightarrow \infty}\left\{\omega_{m, n}\left(r_{1}, r_{2}\right)\right\}^{\frac{1}{m+n}}
$$

$$
M\left(p_{\imath j} ; r_{1}, r_{2}\right)=\max _{\bar{\Gamma}_{r_{1}, r_{2}}}\left|p_{\imath, j}\left(z_{1}, z_{2}\right)\right|
$$

The product set $\left\{p_{m, n}\left(z_{1}, z_{2}\right)\right\}$ of the two set $\left\{p_{m, n}^{(2)}\left(z_{1}, z_{2}\right)\right\}, i=1,2$ where $p_{m, n}\left(z_{1}, z_{2}\right)=$ $\Sigma_{\jmath, k=0}^{m, n} p_{m, n, j, k} z_{1}^{\jmath} z_{2}^{k}$ and $p_{m, n}^{(\imath)^{m, n}}\left(z_{1}, z_{2}\right)=\Sigma_{\jmath, k=0} p_{m, n ; j, k}^{(\imath)} z_{1}^{\jmath} z_{2}^{k}$ if $p_{m, n ; j, k}=\Sigma_{s, t} p_{m, n, s, t}^{(1)} p_{s, t, j, k}^{(2)}$ and is written as follows $\left\{p_{m, n}\left(z_{1}, z_{2}\right)\right\}=\left\{p_{m, n}^{(1)}\left(z_{1}, z_{2}\right)\right\}\left\{p_{m, n}^{(2)}\left(z_{1}, z_{2}\right)\right\}$.

To define the Faber regions in the four dimensional space $E^{4}$, let $C^{(k)}, k=1,2$ be Faber curves in the $z_{k}$-planes and suppose that the corresponding Faber transformation is

$$
z_{k}=\phi_{k}\left(t_{k}\right) \equiv t_{k}+\sum_{n=0}^{\infty} \alpha_{n}^{(k)} t_{k}^{-n}
$$

where the functions $\phi_{k}\left(t_{k}\right)$ are regular and one-to-one (c.f. Newens [4]) for $\left|t_{k}\right|>T_{k}$ Therefore, each of the curve $C^{(k)}$ is the map in the $z_{k}$-plane of the circle $\left|t_{k}\right|=\gamma_{k}$ by the above transformation, where $\gamma_{k}>T_{k} \quad$ For $r>T_{k}$, the map of the circle $\left|t_{k}\right|=r$ is the curve $C^{(k)}$ so that $C^{(k)}$ is really $C \gamma_{k}^{(k)}$ Adopting the notation of Breadze [2] we denote by $B_{r_{1}, r_{2}} ; r_{k}>T_{k}$, the product set

$$
B_{r_{1}, r_{2}}=D\left(C_{r_{1}}^{(1)}\right) \times D\left(C_{r_{2}}^{(2)}\right)
$$

and the closure of $B_{r_{1}, r_{2}}$ is denoted by $\bar{B}_{r_{1}, r_{2}}$. (Adopting the familiar notation, if $C$ is a closed curve we denote by $D(C)$ the interior of $C$, the closure of $D(C)$ is denoted by $\bar{D}(C)$.) The sets $B_{r_{1}, r_{2}}$ and $\bar{B}_{r_{1}, r_{2}}$ are called the Faber regions in the space $E^{4}$ of the two complex variables $z_{1}$ and $z_{2}$

Assumıng that $\left\{f_{n}^{(k)}\left(z_{k}\right)\right\}$ is the $\phi_{r}$-base, let $\left\{p_{m, n}\left(z_{1}, z_{2}\right)\right\}$ be a simple set of polynomials which admits the representation

$$
f_{m}^{(1)}\left(z_{1}\right) f_{n}^{(2)}\left(z_{2}\right)=\sum_{i, j=0}^{m, n} \bar{p}_{m, n ; \imath, j} p_{\imath, j}\left(z_{1}, z_{2}\right) ;(m, n \geq 0) .
$$

Then the Cannon sum $\Omega_{m, n}\left(\rho_{1}, \rho_{2}\right)$ and the Cannon function $\Omega\left(\rho_{1}, \rho_{2}\right)$ of the set $\left\{p_{m, n}\left(z_{1}, z_{2}\right)\right\}$ for the region $\bar{B}_{\rho_{1}, \rho_{2}}$ is

$$
\Omega_{m, n}\left(\rho_{1}, \rho_{2}\right)=\rho_{1}^{n} \rho_{2}^{m} \sum_{i, j=0}^{m, n}\left|\bar{p}_{m, n ; \imath, J}\right| M\left(p_{\imath, J} ; \rho_{1}, \rho_{2}\right)
$$

and

$$
\Omega\left(\rho_{1}, \rho_{2}\right)=\limsup _{m+n \rightarrow \infty}\left\{\Omega_{m, n}\left(\rho_{1}, \rho_{2}\right)\right\}^{\frac{1}{m+n}}
$$

where

$$
M\left(p_{\imath, j} ; \rho_{1}, \rho_{2}\right)=\max _{\bar{B}_{\rho_{1}, \rho_{2}}}\left|p_{\imath, j}\left(z_{1}, z_{2}\right)\right|
$$

Effectiveness of simple sets in Faber regions is justified by the following theorem due to Adepoju [1] Theorem 4.33 . 
THEOREM 1.2. The necessary and sufficient condition for the set $\left\{p_{m, n}\left(z_{1}, z_{x}\right)\right\}$ to be effective in the Faber region $\bar{B}_{\rho_{1}, \rho_{2}}$ is that

$$
\Omega\left(\rho_{1}, \rho_{2}\right)=\rho_{1} \rho_{2}
$$

We now state the definition and properties of associated sets for the Faber region $\bar{B}_{r_{1}, r_{2}} ; r_{k}>0$ Actually, if the simple set $\left\{p_{m, n}\left(z_{1}, z_{x}\right)\right\}$ is given by

$$
p_{m, n}\left(z_{1}, z_{x}\right)=\sum_{i, j=0}^{m, n} q_{m, n ;,, j} f_{2}^{(1)}\left(z_{1}\right) f_{j}^{(2)}\left(z_{2}\right) ;(m, n \geq 0),
$$

then the required associated set is the simple set $\left\{q_{m, n}\left(z_{1}, z_{x}\right)\right\}$ constructed as follows

$$
q_{m, n}\left(z_{1}, z_{x}\right)=\sum_{i, j=0}^{m, n} q_{m, n, 2, j} z_{1}^{2} z_{2}^{j} .
$$

It is seen, from (1 13) and (1.14), that

$$
\left\{p_{m, n}\left(z_{1}, z_{x}\right)\right\}=\left\{q_{m, n}\left(z_{1}, z_{x}\right)\right\}\left\{f_{m}^{(1)}\left(z_{1}\right) f_{n}^{(2)}\left(z_{2}\right)\right\},
$$

and if the set $\left\{p_{m, n}\left(z_{1}, z_{x}\right)\right\}$ admits the representation (19), then

$$
z_{1}^{m} z_{2}^{n}=\sum_{i, j=0}^{m, n} \bar{p}_{m, n ;, j} q_{i j}\left(z_{1}, z_{x}\right) ;(m, n \geq 0) .
$$

Finally, if $\Omega\left(r_{1}, r_{2}\right)$ is the Cannon function of the set $\left\{p_{m, n}\left(z_{1}, z_{x}\right)\right\}$ for the Faber region $\bar{B}_{r_{1}, r_{2}}$, as given by (1.10) and (1 11), and if $\lambda\left(r_{1}, r_{2}\right)$ is the Cannon function of the associated set $\left\{q_{m, n}\left(z_{1}, z_{x}\right)\right\}$ for the polycylinder $\bar{\Gamma}_{r_{1}, r_{2}}$, derived from (1 16) as in (14) and (1.6), then according to [1], that

$$
\lambda\left(r_{1}, r_{2}\right)=\Omega\left(r_{1}, r_{2}\right) .
$$

\section{EFFECTIVENESS IN POLYCYLINDERS OF SIMILAR SETS OF \\ POLYNOMIALS OF TWO COMPLEX VARIABLES IN SIMPLE SETS}

Let $\left\{p_{m, n}^{(k)}\left(r_{1}, r_{2}\right)\right\}$ be two basic sets of polynomials of two complex variables and suppose that the set $\left\{p_{m, n}\left(z_{1}, z_{2}\right)\right\}$ is the set similar to the set $\left\{p_{m, n}^{(2)}\left(z_{1}, z_{2}\right)\right\}$ with respect to the set $\left\{p_{m, n}^{(1)}\left(z_{1}, z_{2}\right)\right\}$, then according to [6]

$$
\left\{p_{m, n}\left(z_{1}, z_{2}\right)\right\}=\left\{p_{m, n}^{(1)}\left(z_{1}, z_{2}\right)\right\}\left\{p_{m, n}^{(2)}\left(z_{1}, z_{2}\right)\right\}\left\{\bar{p}_{m, n}^{(1)}\left(z_{1}, z_{2}\right)\right\}
$$

where

$$
p_{m, n}\left(z_{1}, z_{2}\right)=\sum_{i, J} \sum_{h, k} \sum_{s, t} p_{m, n, 2, j}^{(1)} p_{\imath, J ; h, k}^{(2)} \bar{p}_{h, k ; s, t}^{(1)} z_{1}^{s} z_{2}^{t}
$$

Also, we shall consider the normalizing function of the polynomials $\left\{p_{m, n}^{(k)}\left(z_{1}, z_{2}\right)\right\}$, and $\left\{\bar{p}_{m, n}^{(k)}\left(z_{1}, z_{2}\right)\right\}$ for the polycylinder, in the form

$$
\mu^{(k)}\left(r_{1}, r_{2}\right)=\limsup _{m+n \rightarrow \infty}\left\{r_{1}^{n} r_{2}^{m} M\left(p_{m, n}^{(k)} ; r_{1}, r_{2}\right)\right\}^{\frac{1}{m+n}}
$$

and

$$
\bar{\mu}^{(k)}\left(r_{1}, r_{2}\right)=\limsup _{m+n \rightarrow \infty}\left\{r_{1}^{n} r_{2}^{m} M\left(\bar{p}_{m, n}^{(k)} ; r_{1}, r_{2}\right)\right\}^{\frac{1}{m+n}}
$$

Write $\left\{\bar{p}_{m, n}^{(k)}\left(z_{1}, z_{2}\right)\right\},\left\{\widetilde{p}_{m, n}^{(k)}\left(z_{1}, z_{2}\right)\right\}$ and $\left\{\widehat{p}_{m, n}^{(k)}\left(z_{1}, z_{2}\right)\right\}$ for the inverse, transposed and transposed inverse of the sets $\left\{p_{m, n}^{(k)}\left(z_{1}, z_{2}\right)\right\}$.

Our first result is the following: 
THEOREM 2.1. Let $\left\{p_{m, n}\left(z_{1}, z_{2}\right)\right\}$ be the similar sets of polynomials as given in (21), then, in view of the notations of $(22)$ and $(23)$ we have the following inequality

$$
\begin{aligned}
\lambda\left(r_{1}, r_{2}\right) \leq \frac{\bar{\mu}^{(1)}\left(r_{1}, r_{2}\right)}{\phi_{1}\left(\frac{\bar{\mu}^{(1)}\left(r_{1}, r_{2}\right)}{r_{2}}, \frac{\bar{\mu}^{(1)}\left(r_{1}, r_{2}\right)}{r_{1}}\right)} \mu^{(1)}\left\{\frac{r_{1}}{\bar{\mu}^{(1)}\left(r_{1}, r_{2}\right)} \phi_{1}\left(\frac{\bar{\mu}^{(1)}\left(r_{1}, r_{2}\right)}{r_{2}}, \frac{\bar{\mu}^{(1)}\left(r_{1}, r_{2}\right)}{r_{1}}\right),\right. \\
\left.\frac{r_{2}}{\bar{\mu}^{(1)}\left(r_{1}, r_{2}\right)} \phi_{1}\left(\frac{\bar{\mu}^{(1)}\left(r_{1}, r_{2}\right)}{r_{2}}, \frac{\bar{\mu}^{(1)}\left(r_{1}, r_{2}\right)}{r_{1}}\right)\right\}
\end{aligned}
$$

where

and

$$
\phi_{1}\left(r_{1}, r_{2}\right)=\frac{1}{\phi\left(r_{1}, r_{2}\right)} \bar{\mu}^{(2)}\left\{r_{1} \phi\left(r_{1}, r_{2}\right), r_{2} \phi\left(r_{1}, r_{2}\right)\right\}
$$

$$
\phi\left(r_{1}, r_{2}\right)=\frac{1}{\mu^{(2)}\left(r_{1}, r_{2}\right)} \lambda^{(1)}\left\{\frac{\mu^{(2)}\left(r_{1}, r_{2}\right)}{r_{2}}, \frac{\mu^{(2)}\left(r_{1}, r_{2}\right)}{r_{1}}\right\} .
$$

PROOF. If the right hand side of $(24)$ is infinite there is nothing to prove. If it is finite, let $\rho_{1}, \rho_{1}^{\prime}$, $\rho_{2}, \rho_{2}^{\prime}$ be positive numbers, chosen such that

$$
\left.\begin{array}{l}
\frac{r_{1}}{\overline{\bar{\mu}}^{(1)}\left(r_{1}, r_{2}\right)} \phi_{1}\left(\frac{\bar{\mu}^{(1)}\left(r_{1}, r_{2}\right)}{r_{2}}, \frac{\bar{\mu}^{(1)}\left(r_{1}, r_{2}\right)}{r_{1}}\right)<\rho_{1}<\rho_{1}^{\prime} \\
\frac{r_{2}}{\overline{\bar{\mu}}^{(1)}\left(r_{1}, r_{2}\right)} \phi_{1}\left(\frac{\bar{\mu}^{(1)}\left(r_{1}, r_{2}\right)}{r_{2}}, \frac{\bar{\mu}^{(1)}\left(r_{1}, r_{2}\right)}{r_{1}}\right)<\rho_{2}<\rho_{2}^{\prime}
\end{array}\right\} .
$$

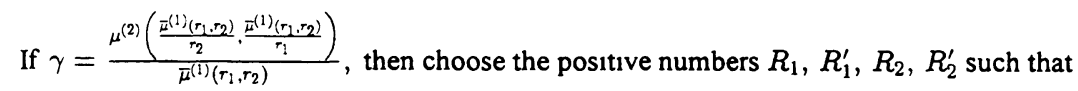

$$
\left.\begin{array}{c}
\frac{1}{r_{2} \gamma} \lambda^{(1)}\left(r_{1} \gamma, r_{2} \gamma\right)<R_{1}<R_{1}^{\prime} \\
\frac{1}{r_{1} \gamma} \lambda^{(1)}\left(r_{1} \gamma, r_{2} \gamma\right)<R_{2}<R_{2}^{\prime}
\end{array}\right\} .
$$

Take the numbers $\tau_{1}, \tau_{1}^{\prime}, \tau_{2}, \tau_{2}^{\prime}$ satısfying the inequalities

$$
\left.\begin{array}{l}
r_{1} \gamma<\tau_{1}<\tau_{1}^{\prime} \\
r_{2} \gamma<\tau_{2}<\tau_{2}^{\prime}
\end{array}\right\}
$$

and the numbers $\eta_{1}<\eta_{1}^{\prime}$ and $\eta_{2}<\eta_{2}^{\prime}$ such that

$$
\bar{\mu}^{(1)}\left(r_{1}, r_{2}\right)<\delta, \quad \delta<\left(r_{2} \eta_{1}, r_{1} \eta_{2}\right) .
$$

Then from (26), (2 7) and (28) we have the following

$$
\mu^{(2)}\left(\eta_{1}, \eta_{2}\right)<\tau, \quad \tau<\left(\tau_{1} \eta_{2}^{\prime}, \tau_{2} \eta_{1}^{\prime}\right)
$$

and from (2 5), (2 6) and (2 7) yrelds

$$
\lambda^{(1)}\left(\tau_{1}, \tau_{2}\right)<R_{1}^{\prime} \tau_{2}^{\prime}, \quad \lambda^{(1)}\left(\tau_{1}, \tau_{2}\right)<R_{2}^{\prime} \tau_{1}^{\prime},
$$

and from (2 5) and (2.6) we get

$$
\bar{\mu}^{(2)}\left(R_{1}, R_{2}\right)<\rho, \quad \rho<\left(\rho_{1}^{\prime} R_{2}^{\prime}, \rho_{2}^{\prime} R_{1}^{\prime}\right) .
$$

Then from (2.3), (2.8), (29) and (2.11) we get

and

$$
\begin{aligned}
& M\left(\bar{p}_{m, n}^{(1)} ; r_{1}, r_{2}\right)<K \frac{\delta^{m+n}}{r_{1}^{n} r_{2}^{m}}, \\
& M\left(p_{m, n}^{(2)} ; \eta_{1}, \eta_{2}\right)<K \frac{\tau^{m+n}}{\eta_{1}^{n} \eta_{2}^{m}},
\end{aligned}
$$




$$
M\left(\bar{p}_{m, n}^{(2)} ; R_{1}, R_{2}\right)<K \frac{\rho^{m+n}}{R_{1}^{n} R_{2}^{m}} .
$$

Using (2 13) and Cauchy's inequality we get

$$
\begin{aligned}
M\left(\widetilde{p}_{h, k}^{(2)} ; \frac{1}{\tau_{1}}, \frac{1}{\tau_{2}}\right) & \leq \sum_{m, n}\left|p_{m, n, h, k}^{(2)}\right|\left(\frac{1}{\tau_{1}}\right)^{m}\left(\frac{1}{\tau_{2}}\right)^{n} \\
& \leq \sum_{m, n} \frac{M\left(p_{m, n}^{(2)} ; \eta_{1}, \eta_{2}\right)}{\eta_{1}^{k} \eta_{2}^{k}}\left(\frac{1}{\tau_{1}}\right)^{m}\left(\frac{1}{\tau_{2}}\right)^{n} \\
& <\frac{K}{\eta_{1}^{k} \eta_{2}^{k}} \sum_{m, n} \frac{\tau^{m+n}}{\eta_{1}^{n} \eta_{2}^{m} \tau_{1}^{m} \tau_{2}^{n}}<\frac{K}{\eta_{1}^{k} \eta_{2}^{k}} .
\end{aligned}
$$

Also from (2 12) and Cauchy's inequality we have

$$
\begin{aligned}
M\left(\widehat{p}_{h, k}^{(2)} ; \frac{1}{\rho_{1}}, \frac{1}{\rho_{2}}\right) & \leq \sum_{m, n}\left|\bar{p}_{m, n ; h, k}^{(2)}\right|\left(\frac{1}{\rho_{1}}\right)^{m}\left(\frac{1}{\rho_{2}}\right)^{n} \\
& \leq \sum_{m, n} \frac{M\left(\bar{p}_{m, n}^{(2)} ; R_{1}, R_{2}\right)}{R_{1}^{h} R_{2}^{k}}\left(\frac{1}{\rho_{1}}\right)^{m}\left(\frac{1}{\rho_{2}}\right) \\
& <\frac{K}{R_{1}^{h} R_{2}^{k}} \sum_{m, n} \frac{\rho^{m+n}}{R_{1}^{n} R_{2}^{m}}\left(\frac{1}{\rho_{1}}\right)^{m,}\left(\frac{1}{\rho_{2}}\right)^{n}<K \frac{1}{R_{1}^{h} R_{2}^{k}} .
\end{aligned}
$$

From (2 8), (2 15) and Cauchy's inequality we obtain

$$
\begin{aligned}
M\left(p_{m, n} ; r_{1}, r_{2}\right) & \leq \sum_{i, j, h, k, s, t}\left|p_{m, n ; i, j}^{(1)}\right|\left|p_{i, j ; h, k}^{(2)}\right|\left|\bar{p}_{h, k ; s, t}^{(t)}\right| r_{1}^{s} r_{2}^{t} \\
& \leq M\left(p_{m, n}^{(1)} ; \tau_{1}, \tau_{2}\right) \sum_{2, J, h, k} M\left(\widetilde{p}_{h, k}^{(2)} ; \frac{1}{\tau_{1}}, \frac{1}{\tau_{2}}\right) M\left(\bar{p}_{h, k}^{(1)} ; r_{1}, r_{2}\right) \\
& <K M\left(p_{m, n}^{(1)} ; \tau_{1}, \tau_{2}\right) .
\end{aligned}
$$

Inserting (2 10), (2 16) and (2.17) in the Cannon sum $\omega_{m, n}\left(r_{1}, r_{2}\right)$ of the similar set, we obtain

$$
\begin{aligned}
& \omega_{m, n}\left(r_{1}, r_{2}\right)=r_{1}^{n} r_{2}^{m} \sum_{\imath, J, h, k, s, t}\left|p_{m, n, \imath, j}^{(1)}\right|\left|\bar{p}_{\imath, j, h, k}^{(2)}\right|\left|\bar{p}_{h, k, s, t}^{(1)}\right| M\left(p_{s, t} ; r_{1}, r_{2}\right) \\
& \leq r_{1}^{n} r_{2}^{m} M\left(p_{m, n}^{(1)} ; \rho_{1}, \rho_{2}\right) \sum_{h, k} M\left(\hat{p}_{h, k}^{(2)} ; \frac{1}{\rho_{1}}, \frac{1}{\rho_{2}}\right) \frac{\lambda_{h, k}^{(1)}\left(\tau_{1}, \tau_{2}\right)}{\tau_{1}^{k} \tau_{2}^{h}} \\
& <K r_{1}^{n} r_{2}^{m} M\left(p_{m, n}^{(1)} ; \rho_{1}, \rho_{2}\right)=K\left(\frac{r_{1}}{\rho_{1}}\right)^{n}\left(\frac{r_{2}}{\rho_{2}}\right)^{m} \rho_{1}^{n} \rho_{2}^{m} M\left(p_{m, n}^{(1)} ; \rho_{1}, \rho_{2}\right) \text {. }
\end{aligned}
$$

If $\frac{r_{1}}{\rho_{1}} \leq \frac{r_{2}}{\rho_{2}}$ we have

$$
\omega_{m, n}\left(r_{1}, r_{2}\right)<K\left(\frac{r_{2}}{\rho_{2}}\right)^{m+n} \rho_{1}^{n} \rho_{2}^{m} M\left(p_{m, n}^{(1)} ; \rho_{1}, \rho_{2}\right) .
$$

Taking the limit of its $(m+n)$-th root letting $m+n \rightarrow \infty$ then (2.19) yields

$$
\lambda\left(r_{1}, r_{2}\right) \leq \frac{r_{2}}{\rho_{2}} \mu^{(1)}\left(\rho_{1}, \rho_{2}\right) .
$$

Finally since $\rho_{1}$ and $\rho_{2}$ can be chosen arbitrarily near to

$$
\frac{r_{1}}{\overline{\bar{\mu}}^{(1)}\left(r_{1}, r_{2}\right)} \phi_{1}\left(\frac{\bar{\mu}^{(1)}\left(r_{1}, r_{2}\right)}{r_{2}}, \frac{\bar{\mu}^{(1)}\left(r_{1}, r_{2}\right)}{r_{1}}\right)
$$

and 


$$
\frac{r_{2}}{\bar{\mu}^{(1)}\left(r_{1}, r_{2}\right)} \phi_{1}\left(\frac{\bar{\mu}^{(1)}\left(r_{1}, r_{2}\right)}{r_{2}}, \frac{\bar{\mu}^{(1)}\left(r_{1}, r_{2}\right)}{r_{1}}\right)
$$

respectively, the required inequality (2.4) of the theorem can be deduced from (2.20) The same result can be arrived at when $\frac{r_{2}}{\rho_{2}} \leq \frac{r_{1}}{\rho_{1}}$ and the theorem is therefore established.

The following corollaries are a direct consequence of the above theorem

COROLLARY 2.1. If the set $\left\{p_{m, n}^{(k)}\left(z_{1}, z_{2}\right)\right\}$ are simple and if the inner set $\left\{p_{m, n}^{(2)}\left(z_{1}, z_{2}\right)\right\}$ is absolute monic (the leading coefficient in the simple set is equal to one) and effective in $\bar{\Gamma}_{r_{1}, r_{2}}$, then, for the similar set $\left\{p_{m, n}\left(z_{1}, z_{2}\right)\right\}$, we have

$$
\lambda\left(r_{1}, r_{2}\right) \leq \frac{\bar{\mu}^{(1)}\left(r_{1}, r_{2}\right)}{\lambda^{(1)}\left(\gamma_{1}, \gamma_{2}\right)} \mu^{(1)}\left\{\frac{\lambda^{(1)}\left(\gamma_{1}, \gamma_{2}\right)}{\gamma_{2}}, \frac{\lambda^{(1)}\left(\gamma_{1} \gamma_{2}\right)}{\gamma_{1}}\right\}
$$

where $\gamma_{1}=\frac{\bar{\mu}^{(1)}\left(r_{1}, r_{2}\right)}{r_{2}}$ and $\gamma_{2}=\frac{\bar{\mu}^{(1)}\left(r_{1}, r_{2}\right)}{r_{1}}$

PROOF. Since $\left\{p_{m, n}^{(2)}\left(z_{1}, z_{2}\right)\right\}$ is a simple monic set of polynomials which is effective in $\bar{\Gamma}_{r_{1}, r_{2}}$ then

$$
\mu^{(2)}\left(r_{1}, r_{2}\right)=r_{1} r_{2} .
$$

Hence, from the inequality (2.4) it follows that

$$
\lambda\left(r_{1}, r_{2}\right) \leq \frac{\bar{\mu}^{(1)}\left(r_{1}, r_{2}\right)}{\lambda^{(1)}\left(\gamma_{1}, \gamma_{2}\right)} \mu^{(1)}\left\{\frac{\lambda^{(1)}\left(\gamma_{1}, \gamma_{2}\right)}{\gamma_{2}}, \frac{\lambda^{(1)}\left(\gamma_{1}, \gamma_{2}\right)}{\gamma_{1}}\right\} .
$$

COROLLARY 2.2. If the set $\left\{p_{m, n}^{(k)}\left(z_{1}, z_{2}\right)\right\}$ are simple monic sets and the two sets effective in $\bar{\Gamma}_{r_{1}, r_{2}}$, then the Cannon function of the similar set satisfies the following equality

$$
\lambda\left(r_{1}, r_{2}\right)=\lambda^{(1)}\left(r_{1}, r_{2}\right) .
$$

PROOF. Since $\left\{p_{m, n}^{(k)}\left(z_{1}, z_{2}\right)\right\}$ are simple monic sets of polynomials and effective in $\bar{\Gamma}_{r_{1}, r_{2}}$, then

$$
\mu^{(1)}\left(r_{1}, r_{2}\right)=r_{1}, r_{2} \quad \text { and } \quad \mu^{(2)}\left(r_{1}, r_{2}\right)=r_{1}, r_{2} .
$$

Inserting these equations in (2 23) we obtain

$$
\lambda\left(r_{1}, r_{2}\right) \leq \lambda^{(1)}\left(r_{1}, r_{2}\right) .
$$

On the other hand, from (2.1) we infer that

$$
\left\{p_{m, n}^{(1)}\left(z_{1}, z_{2}\right)\right\}=\left\{p_{m, n}\left(z_{1}, z_{2}\right)\right\}\left\{p_{m, n}^{(1)}\left(z_{1}, z_{2}\right)\right\}\left\{\bar{p}_{m, n}^{(2)}\left(z_{1}, z_{2}\right)\right\}
$$

and since $\left\{\bar{p}_{m, n}^{(2)}\left(z_{1}, z_{2}\right)\right\}$ is simple monic and effective in $\bar{\Gamma}_{r_{1}, r_{2}}$ we can apply (2 26) to obtain

$$
\lambda^{(1)}\left(r_{1}, r_{2}\right) \leq \lambda\left(r_{1}, r_{2}\right)
$$

Then required equation (2.24) follows from (2.26) and (2.28) and the Corollary is proved.

\section{EFFECTIVENESS OF THE SIMILAR SET OF POLYNOMIALS OF TWO COMPLEX VARIABLES IN FABER REGIONS IN SIMPLE SETS}

We propose, in the present article, to investigate the extension, to two variable case, of the result of Sayyed and Metwally [5] concerning the similar set of simple sets of polynomials of a single variable, being effective in a Faber region. The first result obtaned in this connection is formulated in Theorem 3.1 below, and it gives an exact value of the Cannon function of the similar set.

THEOREM 3.1. Let $\left\{p_{m, n}^{(k)}\left(z_{1}, z_{2}\right)\right\}$ be two simple monic sets of polynomials of two complex variables which are effective in the Faber region $\bar{B}_{\gamma_{1}, \gamma_{2}}$. Then the Cannon function of the similar set $\left\{p_{m, n}\left(z_{1}, z_{2}\right)\right\}$ given by 
for $\bar{B}_{\gamma_{1}, \gamma_{2}}$ is

$$
\left\{p_{m, n}\left(z_{1}, z_{2}\right)\right\}=\left\{p_{m, n}^{(1)}\left(z_{1}, z_{2}\right)\right\}\left\{p_{m, n}^{(2)}\left(z_{1}, z_{2}\right)\right\}\left\{\bar{p}_{m, n}^{(1)}\left(z_{1}, z_{2}\right)\right\}
$$

$$
\Omega\left(\gamma_{1}, \gamma_{2}\right)=\lambda^{(1)}\left(\gamma_{1}, \gamma_{2}\right) \text {, }
$$

where $\lambda^{(1)}\left(\gamma_{1}, \gamma_{2}\right)$ is the Cannon function of the set $\left\{p_{m, n}^{(1)}\left(z_{1}, z_{2}\right)\right\}$ for the polycylinder $\bar{\Gamma}_{\gamma_{1}, \gamma_{2}}$

PROOF. Suppose that $\left\{q_{m, n}\left(z_{1}, z_{2}\right)\right\}$ and $\left\{q_{m, n}^{(1)}\left(z_{1}, z_{2}\right)\right\}$ are the associated sets of the respective sets $p_{m, n}\left\{\left(z_{1}, z_{2}\right)\right\}$ and $\left\{p_{m, n}^{(1)}\left(z_{1}, z_{2}\right)\right\}$ Then from (114) and (31), we deduce tat

$$
\left\{q_{m, n}\left(z_{1}, z_{2}\right)\right\}=\left\{p_{m, n}^{(1)}\left(z_{1}, z_{2}\right)\right\}\left\{p_{m, n}^{(2)}\left(z_{1}, z_{2}\right)\right\}\left\{\bar{q}_{m, n}^{(1)}\left(z_{1}, z_{2}\right)\right\}
$$

Since the simple set $\left\{p_{m, n}^{(1)}\left(z_{1}, z_{2}\right)\right\}$ is absolutely monic and effective in $\bar{B}_{\gamma_{1}, \gamma_{2}}$, then the inverse set $\left\{\bar{p}_{m, n}^{(1)}\left(z_{1}, z_{2}\right)\right\}$ is also absolutely monic and effective in $\bar{B}_{\gamma_{1}, \gamma_{2}}$ Then the associated set $\left\{\bar{q}_{m, n}^{(1)}\left(z_{1}, z_{2}\right)\right\}$ is simple absolutely monic and effective in $\bar{B}_{\gamma_{1}, \gamma_{2}}$ Therefore, we can apply the relation (2.24) of Corollary 22 , to the similar set (3.3), to obtain

$$
\lambda\left(\gamma_{1}, \gamma_{2}\right)=\lambda^{(1)}\left(\gamma_{1}, \gamma_{2}\right)
$$

where $\lambda\left(\gamma_{1}, \gamma_{2}\right)$ is the Cannon function of the set $\left\{q_{m, n}\left(z_{1}, z_{2}\right)\right\}$ for the polycylinder $\bar{\Gamma}_{\gamma_{1}, \gamma_{2}}$ The required relation (3 2 ) can therefore be derived from (3.4) through the relation (1.17)

Now, in the space $E^{4}$ we write

$$
\alpha_{k}=\sup \left\{\left|\phi_{k}\left(t_{k}\right)\right|:\left|t_{k}\right|=\xi_{k}\right\}
$$

With this notation, the required estimate is formulated in the following theorem

THEOREM 3.2. If $\left\{p_{m, n}^{(k)}\left(z_{1}, z_{2}\right)\right\}$ are effective in the Faber region $\bar{B}_{\xi_{1}, \xi_{2}}$, then

$$
\Omega\left(\gamma_{1}, \gamma_{2}\right) \leq \max \left\{\alpha_{1} \gamma_{2}, \alpha_{2} \gamma_{1}\right\}
$$

PROOF. From the definition (3.5) of $\alpha_{k}$ we may infer that

$$
\bar{\Gamma}_{\gamma_{1}, \gamma_{2}} \subset \bar{B}_{\xi_{1}, \xi_{2}} \subset \bar{\Gamma}_{\alpha_{1}, \alpha_{2}}
$$

where

$$
\xi=\max \{|t|:|\phi(t)|=\gamma\} \quad \text { and } \quad \xi>\gamma \quad \text { c.f Newens }[4, \text { p. 188, 1.1.14-26] }
$$

Since the set $\left\{p_{m, n}^{(1)}\left(z_{1}, z_{2}\right)\right\}$ is effective in $\bar{B}_{\xi_{1} \xi_{2}}$, then an appeal to the relation (3.7) leads to the conclusion that the same set is effective in $\bar{\Gamma}_{\gamma_{1}, \gamma_{2}}$, for the class of functions regular in $\bar{\Gamma}_{\alpha_{1}, \alpha_{2}}$ According to Theorem 184 in [1] we can deduce that

$$
\lambda^{(1)}\left(\gamma_{1}, \gamma_{2}\right) \leq \max \left(\alpha_{1} \gamma_{2}, \alpha_{2} \gamma_{1}\right)
$$

and the required inequality (3.6) follows from the equation (3.2)

\section{REFERENCES}

[1] ADEPOJU, J.A., Basic Sets of Goncarov Polynomials and Faber Regions, Ph.D. Thesis (Lagos, Nigeria) (1979).

[2] BREADZE, A.I., The Representation of Analytic Functions of Several Complex Variables in Faber Regions, Sakharthveles SSR Mecnieretalhe Akademas Moambe, 53 (1969), 533-536.

[3] MURSI, M and MAKAR, BUSHRA H., Basic Sets of Polynomials of Several Complex Variables I, Proceedings of the Second Arab Sctence Congress (Cairo) (19957), 51-60.

[4] NEWNS, W.F., On the Representations of Analytic Functions by Infinite Series, Philosophical Transactions of the Royal Society of London, Ser. A, 245 (1953), 424-486.

[5] SAYYED, K.A.M. and METWALLY, M.S., (p-q)-order and (p,q)-type of Basic Sets of Polynomials of Two Complex Variables, Complex Vanables, 26 (1994), 63-68.

[6] SAYYED, K A.M and METWALLY, M.S., Effectiveness of Similar and Inverse Similar Sets of Polynomials in Faber Regions, Sohag Pure and Appl. Sci. Bull., Fac Sc1, Egypt, 9 (1993), 37-49 


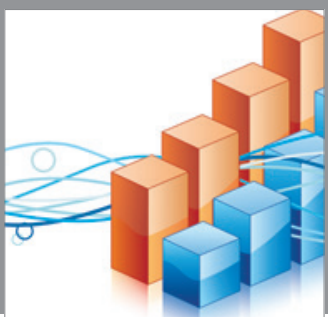

Advances in

Operations Research

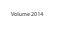

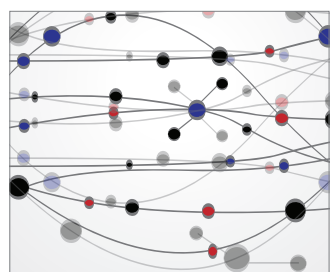

\section{The Scientific} World Journal
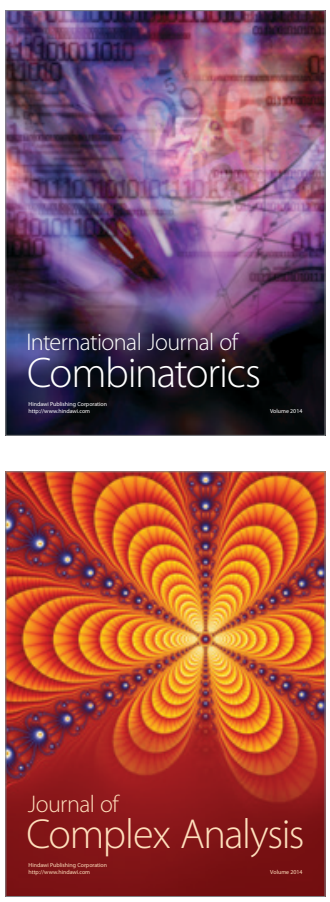

International Journal of

Mathematics and

Mathematical

Sciences
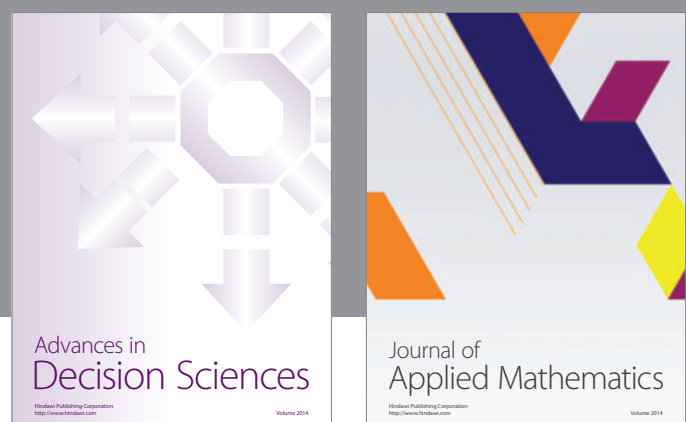

Journal of

Applied Mathematics
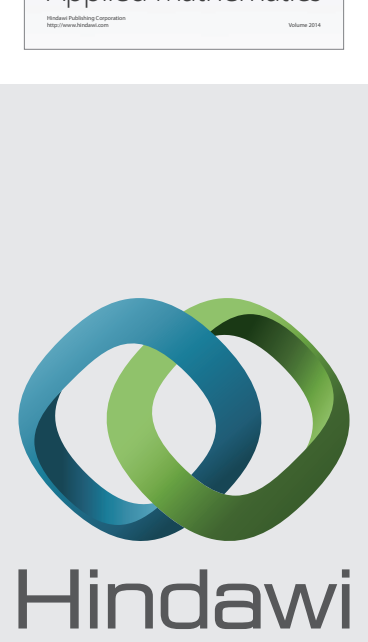

Submit your manuscripts at http://www.hindawi.com
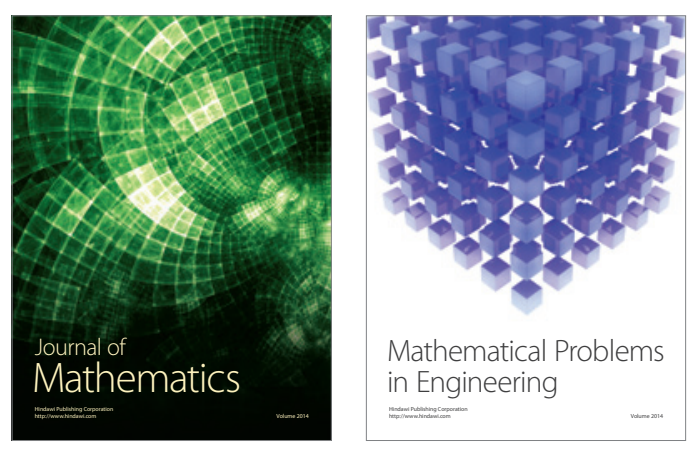

Mathematical Problems in Engineering
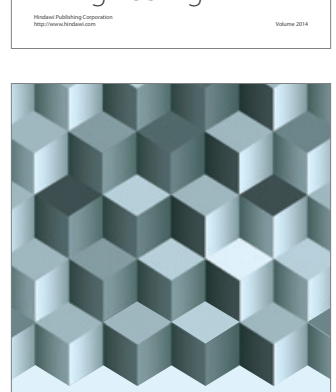

Journal of

Function Spaces
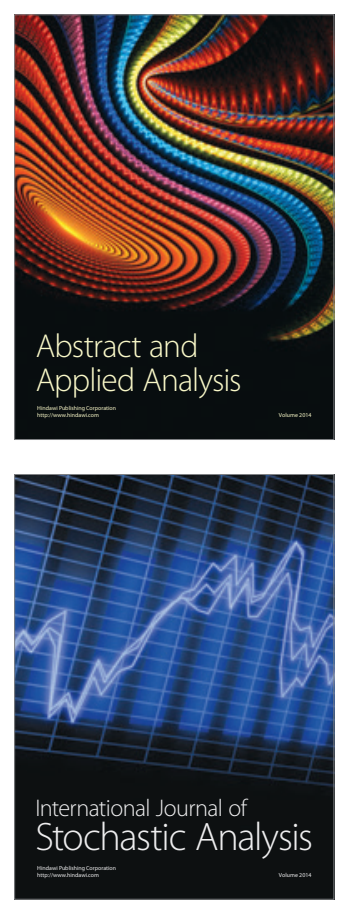

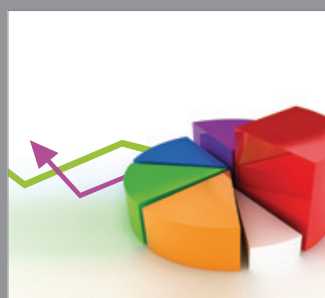

ournal of

Probability and Statistics

Promensencen
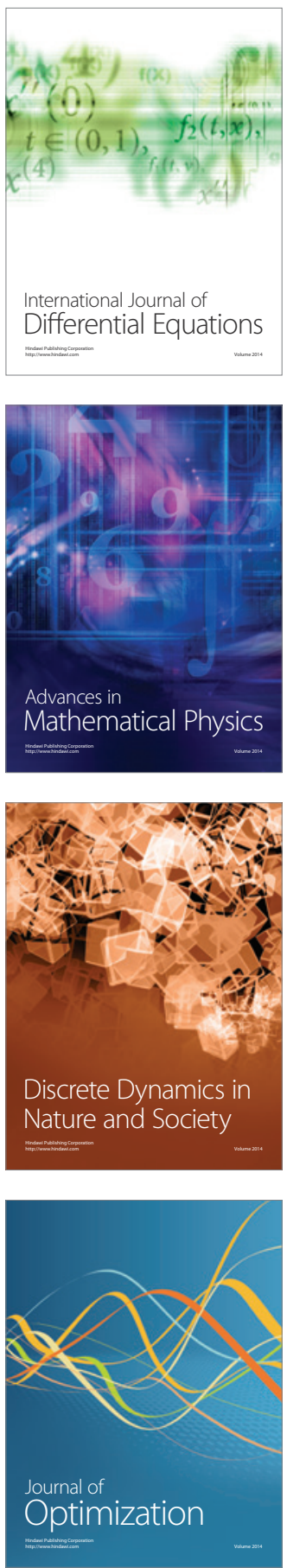